\title{
In vitro Maturation of Oocytes in High Altitude Women with Poly- cystic Ovaries
}

\author{
Luis A. Vargas Tominaga ${ }^{1}$, Ricardo E. Pella Cáceres $^{1}$, Jose A. Vargas Lechuga ${ }^{1}$, Livia S. Bartolo Durán ${ }^{1}$, Mariela \\ Serrano Vargas ${ }^{1}$ \\ ${ }^{1}$ Centro de Fertilidad y Ginecología del Sur, Cusco, Peru
}

Presented as a poster in the Cusco Regional Hospital Golden Anniversary Congress - Cusco, Peru - May, 2014.

\begin{abstract}
Objective: Determine the effectiveness of in vitro maturation of oocytes in the infertility treatment in high altitude women with polycystic ovaries.

Methods: Design: descriptive and retrospective study. Participants: Women with polycystic ovaries and infertility. Interventions: there were 11 women from locations above 7,546 feet above sea level with polycystic ovaries and infertility in which were performed in vitro maturation of oocytes, followed by intracytoplasmic sperm injection, culture and embryo vitrification. After that, the endometrium was prepared and the embryos were thawed and transferred. Main results mesurements: oocytes maturation, fertilization, clinical pregnancy and implantation rates.

Results: Oocytes maturation rate was $86.1 \%$; fertilization rate $90.3 \%$; clinical pregnancy rate $36.4 \%$ and implantation rate $17.4 \%$.

Conclusions: In vitro maturation of oocytes is an effective technique in the infertility treatment of high altitude women with polycystic ovaries.
\end{abstract}

Keywords: In vitro maturation of oocytes, IVM, Polycystic ovaries, PCOS, IVF, ICSI.

\section{INTRODUCTION}

Polycystic ovaries (PO) and the polycystic ovary syndrome (PCOS) are frequent conditions in general population, such as in the group of patients who are treated for infertility. PCOS was first described in 1935 by Irvine Stein and Michel Leventhal through a report of seven patients: four of them were obese, with amenorrea and big ovaries with polycystic appearance. They performed a wedge resection in the seven patients, achieving ovulation in all of them and pregnancy in two (Pacheco, 2008).

While the term "polycystic ovary" is referred to the aspect of the ovary in the ultrasound, the PCOS is diagnosed when this aspect in the ultrasound is associated to symptoms and signs of hyperandrogenism or ovarian dysfunction (Rotterdam ESHRE/ASRM-Sponsored PCOS Consensus Workshop Group, 2004; Pacheco, 2008). In Peru, Noriega (1989) finds PO in $28.8 \%$ of the 104 volunteer women. It even reports PCOS characteristics in the group, such as menstrual irregularities in $50 \%$, overweight in $17 \%$ and hirsutism in the $76 \%$. Inside an infertitlity patient group, Gasco (1992) reports that $22.4 \%$ of the causes are by an endocrine ovarian factor and inside of it, $31.9 \%$ has chronic anovulation and $18.8 \%$ has PCOS. In Centro de Fertilidad y Ginecología del Sur (CFGS), $18.9 \%$ of the patients who requiere in vitro fertilization (IVF) have PO.

In women with $\mathrm{PO}$, the stimulation with gonadotropins, necessary for IVF procedures, can generate excessive response with ovarian hyperstimulation syndrome with hemodynamic instability and life risk for the patient. It can also generate a poor response with few mature oocytes and poor quality. On the other side, those patients with appropiate ovarian response, may have low implantation rates due to high estrogen level and deleterious effects in the endometrial receptivity. Against these risks that produces ovarian stimulation in PO patients, in vitro maturation (IVM) of oocytes is constituted as an alternative technique.

Peru has a big geographic diversity, with populated centers, both at sea level and high altitudes. Around 30\% of peruvian population lives above 9,842 feet above sea level (F.A.S.L). Menarche takes longer, and menopause happens earlier, because of a high altitude factor and not because of racial, nutritional, or socioeconomic differences, shortening the reproductive life of the andean woman (Reynafarje, 1990). The pathologies related to infertility are probably similar at sea level and at high altitude. However, earliest decrease of the ovarian reserve is an additional factor that motivates the realization of IVF procedures at high altitude.

IVM of oocytes consists in obtaining inmature oocytes (in germinal vesicle state) from antral ovarian follicles without or minimally stimulated with gonadotropins before being matured under laboratory conditions in culture supplemented with hormones. This technique allows us to obtain oocytes in metaphase II in order to be able to be fertilized without needing elevated gonadotropin doses.

IVM of oocytes was proposed by Pincus and Enzmann in 1935 (Pincus \& Enzmann, 1935) and then by Edwards, in 1969 (Edwards et al., 1969). In 1991, the first pregnancy product of IVM of oocytes was achieved (Cha et al., 1991), and now there are more than 2,000 children achieved through this technique. The first peruvian child product of IVM of oocytes was born in 2012, whose mother required such technique because of anovulation associated with amenorrhea and anorexia (Vargas et al., 2012a; Vargas et al., 2012b).

The present study seeks to determine the effectiveness of IVM of oocytes in the infertility treatment in high altitude women with PO.

\section{MATERIAL AND METHOD}

It is a retrospective and descriptive study of 11 women with PO and infertility diagnosis in whom IVM was carried out, from June 2011 to October 2013. The procedures were done in the Embryology Laboratory of the CFGS, in the city of Cusco, at 10,925 F.A.S.L Patients were from regions of Cusco, Apurimac and Puno (locations above 7,546 F.A.S.L).

It is defined as PO to a number of antral follicles (AFC) of 12 or more (follicles between 2 and $9 \mathrm{~mm}$ ) in one or both ovaries (Pacheco, 2008; Rotterdam ESHRE/ASRM-Sponsored PCOS Consensus Workshop Group, 2004). The characteristics of the patients are described in Table 1.

75 to $150 \mathrm{IU}$ of rFSH for 3 consecutives days were administrated between the first and fifth day of the cycle. Human chorionic gonadotropin (hCG) was not administrated. Between the sixth and eighth day of the cycle follicular as- 
Table 1. Characteristics of the patients

\begin{tabular}{|l|l|l|}
\hline \multicolumn{2}{|l|}{ Characteristics } & Media \\
\hline Age & $27-39$ years old & 33.6 \\
\hline AFC & $19-36$ & 22.4 \\
\hline Diagnosis & $\begin{array}{l}\text { Anovulation (4), Male fa- } \\
\text { tor(4), } \\
\text { Unexplained infertility (2), } \\
\text { Endometriosis (1) }\end{array}$ & \\
\hline
\end{tabular}

*AFC: Antral follicle count

piration under intravenous sedation was carried out using an 18 gauge needle of single lumen (Vitrolife) or inmature oocytes aspiration needle (Cook).

The cumulus-oocytes complex (COC) (Fig. 1A) were cultivated in maturation medium with $75 \mathrm{mIU} / \mathrm{ml}$ of $\mathrm{rFSH}$ (Gonal-F, Merck-Serono) and $100 \mathrm{mIU} / \mathrm{ml}$ of hCG (Pregnyl, Schering-Plough or Choragon, Ferring), supplemented with $10 \%$ of inactivated patient's serum. After 36 hours, the COC were evaluated (Fig. 1B) and metafase II oocytes were inseminated by intracytoplasmic sperm injection (ICSI) (Fig. 1C) and cultivated in Global medium (LifeGlobal). 18 hours afer ICSI was carried out, fertilization of the oocytes was evaluated (Fig. 1D), and these were kept in the same medium for the next 24 hours (Fig. 1E).

Since there is a mismatch between embryonic and endometrial development, embryo freezing by vitrification was performed (Kuwayama et al., 2005) using McGill Cryoleaf $^{\mathrm{TM}}$ (Origio) device and were stored in liquid nitrogen.

After 2 to 7 months, at the beginning of menstruation, estradiol valerate (Progynova) was used in ascendent progresive doses orally from $2 \mathrm{mg}$ to $12 \mathrm{mg}$ daily until having an endometrial thickness above $8 \mathrm{~mm}$. $200 \mathrm{mg}$ of micronized progesterone (Utrogestan, Ferring or Geslutin PNM, Tecnofarma) and $90 \mathrm{mg}$ of progesterone gel (Crinone $8 \%$, Merk-Serono) were added daily, both intravaginal, or the association of $600 \mathrm{mg}$ of micronized progesterone plus the application of the progesterone vaginal ring (Fertiring, ABL Pharma).

Embryos were thawed and cultured for 24 hours (Fig. $1 F)$. Afterwards, they were transferred to the uterine cavity using a Frydman Ultrasoft catheter (CCD Laboratories)

Figure 1. Sequence of the process of IVM of oocytes (A) Immature COC. (B) COC at 36 hours of culture with rFSH + hCG + patient's serum. (C) Denuded oocyte showing the second polar body (metaphase II). (D) Oocyte fertilization and evaluation at 16 hours. (E) Embryo after 42 hours of development and freezed by vitrification. (F) Thawed embryo, cultivated for 24 hours and transferred.

(A)

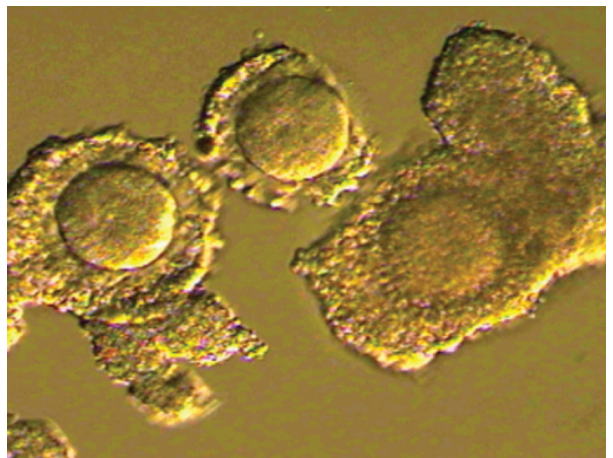

(C)

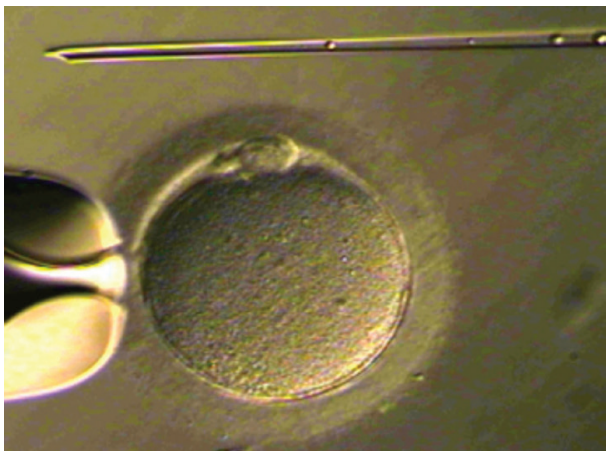

(E)

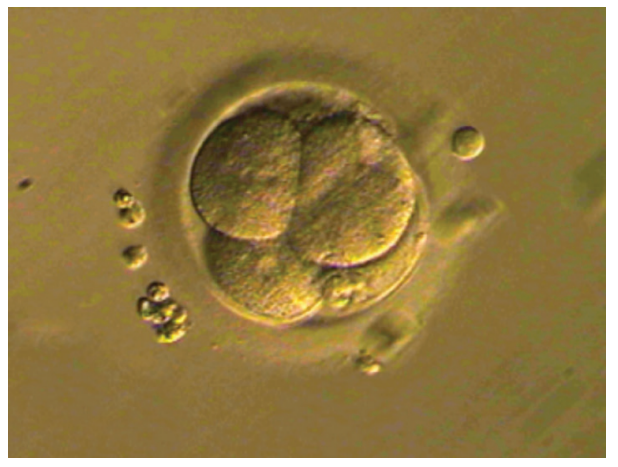

(B)

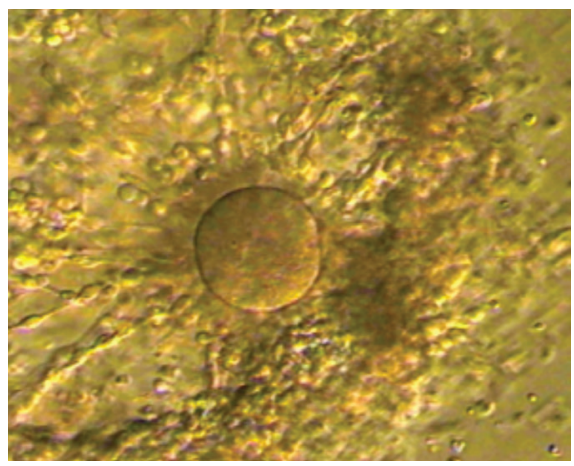

(D)

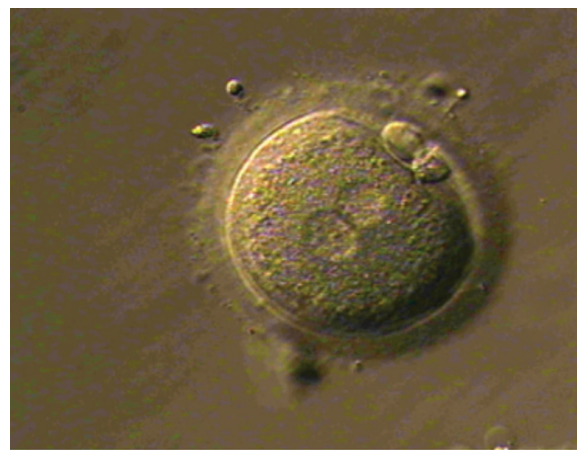

(F)

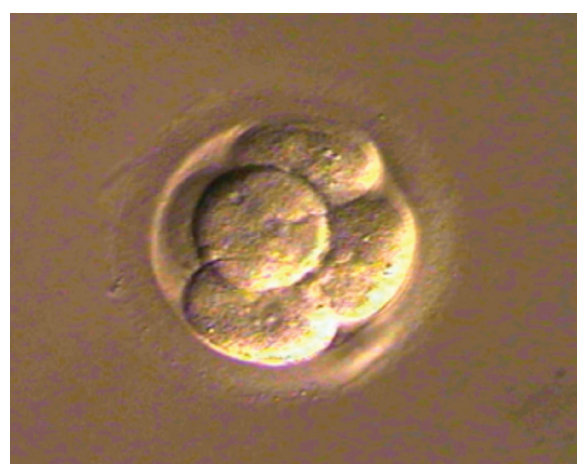


Table 2. Oocyte and embryo development

\begin{tabular}{|l|l|}
\hline Results & $36(1$ to 6 oocytes, 3.3 in average) \\
\hline Aspirated oocytes & 31 (maturation rate: $31 / 36=86.1 \%)$ \\
\hline Oocytes that accomplish maturation & 28 (fertilization rate: $28 / 31=90.3 \%)$ \\
\hline Oocytes that accomplish fertilization & 25 (cleavage rate: $25 / 28=89.3 \%)$ \\
\hline Oocytes that accomplish cleavage & $22(1$ to 3 embryos, 2.1 in average) \\
\hline Transferred embryos & $12(12 / 23=52.2 \%)$ \\
\hline Positive $\boldsymbol{\beta}$-hCG rate & $7 / 11=63.6 \%$ \\
\hline Clinical pregnancy rate & $4 / 11=36.4 \%$ \\
\hline Implantation rate & $4 / 22=17.4 \%$ \\
\hline
\end{tabular}

under abdominal ultrasound guide.

After 13 days of the transfer, dosage of $\beta$-hCG was performed. In case of having a positive result, a vaginal ultrasound was performed 1 or 2 weeks after the test. It is defined as a clinical pregnacy the presence of a gestational sac and presence of an embryo with cardiac activity.

\section{RESULTS}

Table 2 describes oocyte and embryo development.

A patient achieved a fully 38 week pregnancy with the birth of a healthy $3,250 \mathrm{gr}$ girl through elective caesarean section. A second patient required an emergency caesarean section at the 36 week due to pre-eclampsia, having a healthy $2,390 \mathrm{gr}$ girl.

Two patients having ongoing active pregnancy, developed the disappearance of the cardiac embryonic activity in the 8 week. A third patient developed gestational sac without embryo and was considered as a biochemical pregnancy.

\section{DISCUSSION}

In our laboratory, IVM of oocytes achieved high maturation, fertilization and cleavage rates $(86.1 \%, 90.3 \%$ y $89.3 \%$, respectively). International reports show oocyte maturation rates between 33 and $82 \%$, fertilization rates between 44 and $80 \%$ and cleavage rates between 19 and $96 \%$ (Farsi et al. 2013). Despite the limited number of oocytes obtained by aspiration (3.3 in average), clinical pregnancy rate of $36.4 \%$ was achieved, which is between the 17 to $41 \%$ rate that is reported for this technique. Eventhough there are reports that claim an advantage in the administration of hCG before obtaining the oocytes (Son et al., 2008a, 2008b, Tan \& Child, 2002, Chian, 2004) it's being also manifested that hCG interrupts comunication between follicular cells and the oocyte; this is an important event to the adquisition of a good embryonary potential development (Albertini et al., 2001). In our IVM protocol, we do not use hCG in vivo. We mature the oocyte in vitro putting them in the presence of FSH and hCG in the medium and satisfactory results with this technique were found (De Vos et al., 2011). The advantage of combining IVM of oocytes and vitrification of the embryos, is to postpone embryo transfer when the endometrium is sufficiently receptive.

Abortion development in 2 patients with clinical pregnancy, might be asociated to inherent factors, such as polycystic ovaries and age (37 and 38 years old respectively). In CFGS, population that requires assisted reproduction techniques presents a notorious decrease in pregnancy rates from 35 years old (Vargas et al., 2013).

IVM of oocytes provides and effective alternative for high altitude women with PO, obtaining good maturation, fertilization and cleavage rates without using high dose of ovulation inductors. We consider that to improve clinical strategies to achieve more number of collected oocytes is required, and these will allow us to improve clinical pregnancy rates.

\section{CONCLUSIONS}

IVM of oocytes is an effective technique in infertility treatments in high altitude women with PO.

\section{CONFLICT OF INTERESTS}

No conflict of interest have been declared.

\section{Corresponding author:}

Luis Vargas Tominaga

Centro de Fertilidad y Ginecología del Sur (CFGS)

Cuzco - Peru

E-mail: luisvargastominaga@hotmail.com

\section{REFERENCES}

Albertini DF, Combelles CM, Benecchi E, Carabatsos MJ. Cellular basis for paracrine regulation of ovarían follicle development. Reproduction. 2001;121: 647-53.

Cha KY, Koo JJ, Ko JJ, Choi DH, Han SY, Yoon TK. Pregnancy after in vitro fertilization of human follicular oocytes collected from nonstimulated cycles, their culture in vitro and their transfer in a donor oocyte program. Fertil Steril. 1991;55:109-13.

Chian RC. In-vivo maturation of immature oocytes for infertile women with PCOS. Reprod Biomed Online. 2004;8:547-52.

De Vos M, Ortega-Hrepich C, Albuz FK, Guzman L, Polyzos NP, Smitz J, Devroey P. Clinical outcome of non hCG-primed oocyte in vitro maturation treatment in patients with polycystic ovaries and polycyctic ovary syndrome. Fertil Steril. 2011;96:860-4.

Edwards RG, Bavister BD, Steptoe PC. Early stages of fertilization in vitro of human oocytes matured in vitro. Nature. $1969 ; 221: 632-5$.

Farsi MM, Kamali N, Pourghasem M. Embryological aspects of oocyte in vitro maturation. Int J Mol Cell Med. 2013;2:99-109.

Gasco LF. Ovarian-endocrine factor in infertility marriyes. [Thesis]. Universidad Peruana Cayetano Heredia. Lima-Perú. 1992. 
Kuwayama M, Vajta G, Leda S, Kato O. Comparison of open and closed methods for vitrification of human embryos and the elimination of potential contamination. Reprod Biomed Online. 2005;11:608-14.

Noriega LO. Prevalencia del síndrome de ovarios poliquísticos en una población normal [Thesis]. Universidad Peruana Cayetano Heredia. Lima-Peru. 1989.

Pacheco, J. Polycystic ovary syndrome treatment in the infertile woman. Notes on the recent Thessaloniki Consensus. Rev Peru Ginecol Obstet. 2008;54:143-8.

Pincus G, Enzmann EV. Can Mammalian Eggs Undergo Normal Development in Vitro? Proc Natl Acad Sci USA. $1934 ; 20: 121-2$.

Reynafarje C, eds. La adaptación a las grandes alturas. Contribución peruana a su estudio. Lima: Propaceb. 1990.

Rotterdam ESHRE/ASRM-Sponsored PCOS Consensus Workshop Group. Revised 2003 consensus on diagnostic criteria and long-term health risks related to polycystic ovary syndrome. Fertil Steril. 2004;81:19-25.

Son WY, Chung JT, Dermitas E, Holzer H, Sylvestre C, Buckett W, Chian RC, Tan SL. Comparison of in-vitro maturation cycles with and without in-vivo matured oocytes retrieved. Reprod Biomed Online. 2008a;17: 59-67.

Son WY, Chung JT, Herrero B, Dean N, Demirtas E, Holzer $H$, Elizur S, Chian RC, Tan SL. Selection of the optimal day for oocyte retrieval based on the diameter of the dominant follicle in hCG-primed in vitro maturation cycles. Hum Reprod. $2008 b ; 23: 2680-5$.

Tan SL, Child TJ. In vitro maturation of oocytes from unstimulated polycyctic ovaries. Reprod Biomed Online. 2002;4:18-23.

Vargas L, Pella R, Vargas A, Bartolo L. [Pregnancy by oocytes in vitro maturation in amenorrhoeic patient with history of anorexia nervosa]. Rev Peru Ginecol Obstet. 2012a;58:133-6.

Vargas L, Pella R, Vargas A, Bartolo L. [In vitro maturation of oocytes: effective alternative in assisted reproduction] Rev Peru Ginecol Obstet. 2012b;58:263-6.

Vargas L, Pella R, Bartolo L, Vargas A, Vargas A, Pella A. Blastocyst transfer in women over 35 years of age not achieve pregnancy: Experience at 11,000 feet above sea level. Fertil Steril. 2013;100:s500. 